\title{
GCM simulations of Uranus and Neptune: general circulation and eddy activity
}

Gwenaël Milcareck $k^{1,2}$, Sandrine Guerlet ${ }^{1}$, Aymeric Spiga ${ }^{1}$, Jérémy Leconte ${ }^{3}$, Déborah Bardet ${ }^{1}$, Franck Montmessin ${ }^{2}$, and Alexandre Boissinot ${ }^{1}$

${ }^{1}$ CNRS, LMD, Paris, France (gwenael.milcareck@Imd.ipsl.fr)

${ }^{2}$ CNRS, LATMOS, Guyancourt, France (gwenael.milcareck@latmos.ipsl.fr)

${ }^{3}$ CNRS, LAB, Pessac, France

Located at an average distance of $19 \mathrm{AU}$ and $30 \mathrm{AU}$ respectively from the sun, Uranus and Neptune are mysterious and hard-to-reach worlds. These two planets are characterized by low sunshine and long orbital periods and by very marked seasonal variations (especially for Uranus). Today, Voyager 2 is the only probe to have made a flyby of these two planets and it has revealed that these cold worlds have an intense atmospheric circulation $[1,2]$. Since then, other zonal wind measurements using cloud tracking have been carried out with the aid of Hubble Space Telescope and terrestrial observatories like the Keck observatory or the Very Large Telescope (see review by [3] and references therein). These measurements confirm and complement previous Voyager 2 observations: zonal flow over these planets is characterized by two prograde jets at mid-latitudes as well as a large retrograde jet at the equator. While the amplitude of the prograde jets is similar on the two planets ( $250 \mathrm{~m} / \mathrm{s}$ on Uranus, $275 \mathrm{~m} / \mathrm{s}$ on Neptune), that of the equatorial retrograde jet is different: it is $-50 \mathrm{~m} / \mathrm{s}$ on Uranus but reaches $-400 \mathrm{~m} / \mathrm{s}$ on Neptune.

Understanding the origin of these jets is one of the major current challenges in physics of planetary atmospheres. Furthermore, the impact of Uranus and Neptune different radiative forcings on their atmospheric circulation remains to be assessed. The weath of observations has motivated the need to develop models in order to better interpret these observations and to understand the processes that govern their atmospheric circulation.

Several models have attempted to explain the atmospheric circulation visible on the four giant planets. In the case of deep convection models, the equatorial sub-rotation could not be reproduced $[4,5,6,7,8]$ except by greatly modifying the known internal heat flux, which is thus not satisfactory $[9,10]$. In the case of shallow-flow models, this equatorial sub-rotation could be reproduced if convective Rossby wave generation is weak or absent or baroclinic eddies generation is sufficiently strong [11] or by release of latent heat by the water vapor layer located at 200-300 bars [12]. However, the simulated jet speeds are too slow compared to observations. Furthermore, the radiative transfer was idealized as Newtonian cooling, and seasonal effects were ignored. Here, the objective is to apply a shallow-flow model which takes into account realistic physical parameterizations, to attempt to reproduce this circulation, to answer observational questions but also to determine the dynamical processes governing in these atmospheres. 


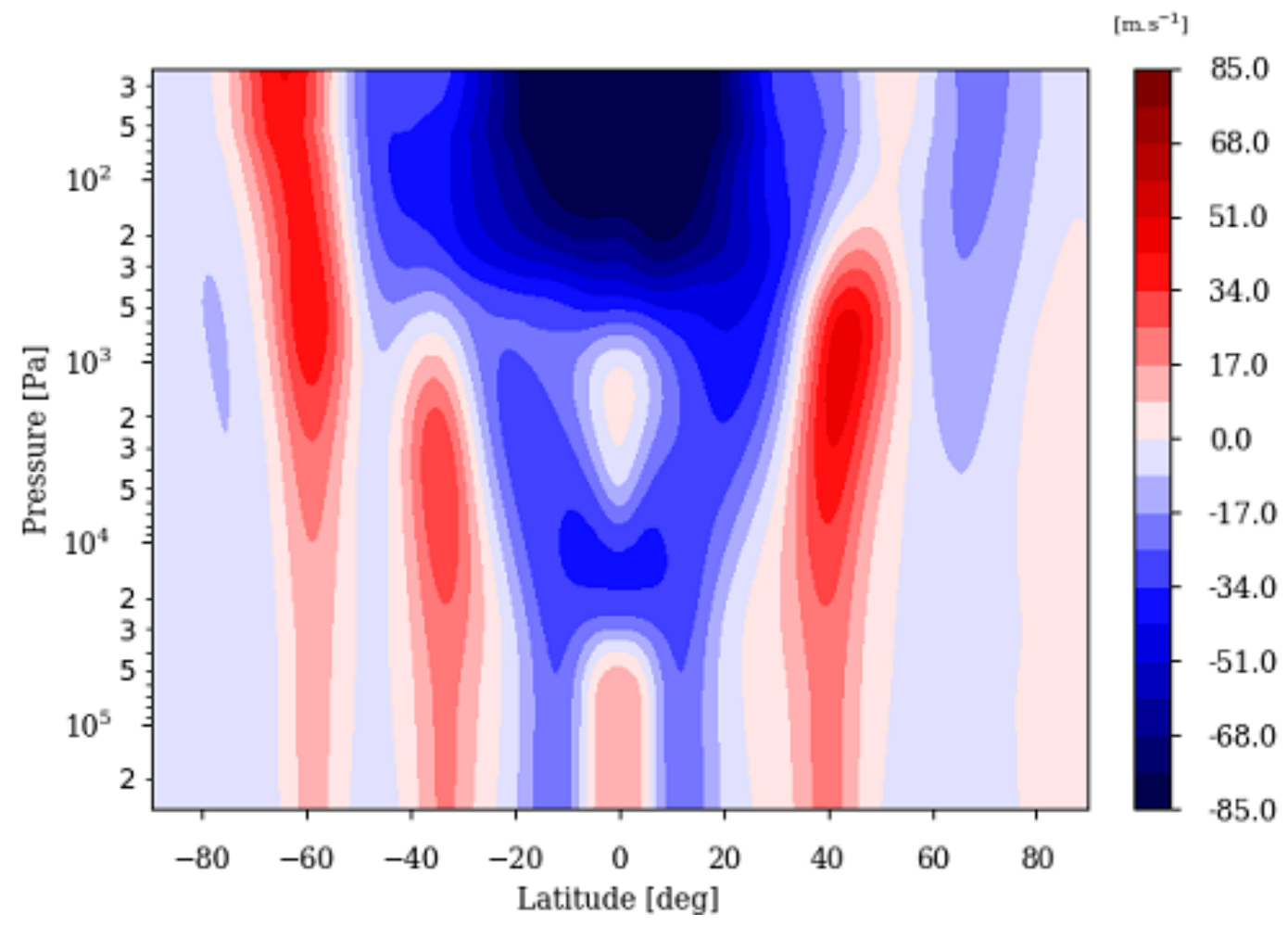

Figure 1: Latitude-pressure cross-section of zonal-mean zonal wind of the third simulated year on Neptune from our preliminary results. A complex zonal structure is present in mid-latitudes and at equator but the zonal structure is still evolving because the radiative-convective equilibrium has still not been reached in the troposphere. Here, the retrograde equatorial stratospheric jet reaches -130 $\mathrm{m} / \mathrm{s}$.

We introduce the DYNAMICO-giant model which is a General Circulation Model (GCM) developed by the Laboratoire de Météorologie Dynamique (LMD), previously used for the atmospheres of giant planets $[13,14,15,16]$. The model is composed of a dynamic core which uses an icosahedral grid [17] and which is coupled to independent physical parameterizations such as a radiative transfer or convection. In this study, the radiative transfer module employs the correlated-k formalism. We take into account gaseous opacities from $\mathrm{CH}_{4}, \mathrm{C}_{2} \mathrm{H}_{2}$ and $\mathrm{C}_{2} \mathrm{H}_{6}$ and collision-induced absorption opacities $\left(\mathrm{H}_{2}-\mathrm{H}_{2}, \mathrm{H}_{2}-\mathrm{He}, \mathrm{H}_{2}-\mathrm{CH}_{4}, \mathrm{He}-\mathrm{CH}_{4}\right.$ and $\left.\mathrm{CH}_{4}-\mathrm{CH}_{4}\right)$ assuming a $\mathrm{H}_{2}$ ortho/para fraction at equilibrium. Stratospheric aerosols [18] and two cloud layers of $\mathrm{CH}_{4}$ and $\mathrm{H}_{2} \mathrm{~S}$ are also included. Our model accounts for the internal heat flux, for multiple scattering as proposed by [19] and Rayleigh scattering.

During this congress, we will discuss the insights gained from GCM simulations at high horizontal resolution (equivalent to $1^{\circ}$ in latitude/longitude) on a layer of the atmosphere located between 3 bar and 0.3 mbar and split in 40 vertical levels. The preliminary results obtained from 3 simulated years on Neptune by the GCM make it possible to highlight a complex zonal circulation characterized by an intense retrograde equatorial jet (fig.1) and a strong eddy activity characterized by bursts. First of all, we will present the zonal and meridional circulation obtained after 10 simulated Neptune years, which is the expected spin-up time based on our experience with Saturn and Jupiter. Then, we will compare the thermal structure obtained on our simulations with the observations from [20]. Next, we will discuss the eddy activity highlighted by the diagnostics of the circulation (eddy momentum transport, spectral analysis of waves,...) and finally, its contribution to the acceleration or deceleration of the simulated jets. 


\section{Acknowledgements}

The authors would like to thank Leigh N. Fletcher and Glenn S. Orton for sending us the zonal mean temperature data on Neptune and Uranus, and Bernard Schmitt for sending us the optical constants of $\mathrm{H}_{2} \mathrm{~S}$ ice. G. Milcareck, S. Guerlet and A. Spiga acknowledge funding from Agence Nationale de la Recherche (ANR) project SOUND, ANR-20-CE49-00009-01.

\section{References}

[1] Lindal et al. (1987). Journal of Geophysics Research, 92.

[2] Lindal et al. (1992). Astronomical Journal, 103.

[3] Fletcher et al. (2020). Space Science Reviews, 216.

[4] Yano et al. (2005). Geophysical and Astrophysical Fluid Dynamics, 99.

[5] Heimpel et al. (2005). Nature, 438.

[6] Vasavada and Showman (2005). Reports on Progress in Physics, 68.

[7] Heimpel and Aurnou (2007). Icarus, 187.

[8] Glatzmaier et al. (2009). Geophysical and Astrophysical Fluid Dynamics, 103.

[9] Aurnou et al. (2007). Icarus, 190.

[10] Soderlund et al. (2013). Icarus, 224.

[11] Liu and Schneider (2010). Journal of Atmospheric Sciences, 67.

[12] Lian and Showman (2010). Icarus, 207.

[13] Guerlet et al. (2014). Icarus, 238.

[14] Spiga et al. (2020). Icarus, 335.

[15] Cabanes et al. (2020). Icarus, 345.

[16] Bardet et al. (2021). Icarus, 354.

[17] Dubos et al. (2015). Geoscientific Model Development, 8.

[18] Vatant d'Ollone et al. In preparation.

[19] Toon et al. (1989). Journal of GeophysicsResearch, 94.

[20] Fletcher et al. (2014). Icarus, 231. 